\title{
EL DEBATE SOBRE LA DURACIÓN DE LA JORNADA DE TRABAJO
}

\author{
María Olaya MarTín RodríGuez \\ Departamento de Derecho del Trabajo y de la Seguridad Social \\ Facultad de Derecho. Universidad Complutense de Madrid \\ olayamr@gmail.com
}

\section{INTRODUCCIÓN}

El propósito de este ensayo radica en abrir un debate sobre la duración de los tiempos de trabajo. Para ello haré referencia a la evolución histórica del tiempo de trabajo. La lucha histórica del movimiento de los trabajadores y del sindicalismo por la reducción de la jornada laboral estuvo íntimamente ligada a las condiciones de trabajo del conjunto asalariado. Esto es, trabajar menos horas como elemento de subsistencia frente a unos periodos larguísimos y extenuantes. Hoy la exigencia sindical de la reducción de los tiempos de trabajo no está relacionada con la subsistencia de la condición de trabajo y de vida, sino con otras hipótesis y objetivos. Ahora la relación que se pretende establecer está en la reducción del tiempo de trabajo y creación de empleo, de un lado, y, de otro, en que las personas logremos un nexo más gratificante entre tiempos de trabajo y tiempos de vida. Pretendo, por tanto, mostrar los cambios que ha sufrido la jornada de trabajo hasta nuestros días y los debates surgidos en torno a la reducción de la jornada de trabajo como medida de reparto y creación de empleo.

Las modificaciones de la economía mundial y el carácter neoliberal han traído cambios en los procesos de trabajo y en la amplitud de los tiempos. También se han precarizado las condiciones de trabajo, de tal forma que si bien es una realidad el aumento del desarrollo técnico y una subida en los niveles de productividad del trabajo, no está directamente relacionado con una mejora de las condiciones de trabajo y la ampliación de las garantías y beneficios en materia de seguridad en el trabajo.

El desarrollo científico y técnico que, en el mejor de los casos, debería servir para liberar a los seres humanos y garantizarles un desarrollo más pleno y equilibrado, actualmente se percibe como una amenaza, pues presupone una reducción de las ofertas de empleo. Aunque, por un lado, 
los teóricos que hablan del fin del trabajo o del no trabajo hablan sobre la muerte prematura del trabajo y de la clase obrera, los autores también mencionan que en la actualidad se trabaja más, durante una mayor cantidad de horas, creciendo el trabajo femenino, infantil y forzado sobre todo en los países en vías de desarrollo ${ }^{1}$. A lo que se añade que la precarización del trabajo y los salarios de muchos obreros obligan a recurrir al doble o triple empleo. La Organización Internacional del Trabajo (OIT) insiste en la necesidad de un trabajo decente y digno para todos e insiste en la incorporación de un análisis ético de la cuestión.

En el caso de la jornada o las horas de trabajo, aun cuando el problema aparente ha sido resuelto y podría parecer parte de la historia, un estudio pormenorizado del tema nos demuestra que el problema de la prolongación de la jornada de trabajo y del aumento de la tensión dentro de las mismas sigue siendo permanente.

Estudios de la OIT y de otros organismos internacionales han apuntado hacia el asunto de las condiciones laborales, en general, y de las horas de trabajo, en particular, en el contexto de la llamada reestructuración del mundo del trabajo alertando sobre la necesidad de lograr un marco justo de análisis que permita el logro de un trabajo decente para todos.

\section{ANÁLISIS HISTÓRICO DE LA JORNADA DE TRABAJO}

El debate sobre la jornada de trabajo y su dimensión temporal data de los tiempos de la Revolución Industrial en Inglaterra en la primera mitad del siglo XIX. En esta etapa los procesos de trabajo y de elaboración de mercancías se desplazaron del ambiente doméstico al fabril, siendo la rama de textiles de algodón la primera en introducir el nuevo sistema fabril, que rápidamente se extendió. La extensión del sistema fabril, que indiscutiblemente fue un signo general del progreso, tuvo una significación muy contradictoria para los trabajadores que se habían librado de la servidumbre feudal y unido al sistema de trabajo asalariado. Ello significaba un cambio en las condiciones de trabajo que significaba una nueva disciplina, reforzamiento de la coacción y deterioro de las condiciones de trabajo en lo refe-

\footnotetext{
${ }^{1}$ La globalización y la economía competitiva entre países lleva a las empresas a descentralizar sus procesos productivos a zonas o países en vía de desarrollo para tener unos costes laborales menores. De tal forma que mientras en algunos países se consiguen ciertas condiciones y mejoras laborales, en otros, por el contrario, aún existe la explotación laboral.
} 
rente a riesgos de accidentalidad, mortalidad y un aumento sin precedentes de la jornada laboral ${ }^{2}$.

En esta etapa eran normales las jornadas de trabajo superiores a doce horas y la explotación del trabajo infantil y femenino. Los accidentes de trabajo e incluso muertes en el trabajo eran frecuentes, lo cual provocó protestas dentro de los trabajadores y otros sectores de la sociedad civil. En Inglaterra se dictaron leyes para regular el trabajo en las fábricas (Factory Acts), pero no fue hasta la Ley de Fábricas de 1874 cuando se estableció una jornada laboral de diez horas al día para todos los tipos de trabajo en Inglaterra.

El tránsito de una fase mercantil a otra industrial dentro del desarrollo capitalista habrá de exigir la creación de un cuerpo normativo integrador que asuma la misión de imponer al conflicto industrial un cauce de circulación compatible con el mantenimiento y desarrollo del modo de producción capitalista. Con arreglo a una cronología variable en función del grado de desarrollo económico de los distintos Estados se inicia durante el siglo XIX la escala de intervencionismo normativo en las relaciones entre el capital y el trabajo dando lugar a las primeras leyes obreras. El Derecho de Trabajo comienza restringiendo el tiempo de trabajo primero en niños y mujeres, y posteriormente se extiende hacia los adultos. En todos los países este proceso será parecido. La mayoría de las veces los Estados acababan imponiendo la limitación de la jornada presionados por los acontecimientos, como forma de acabar con las huelgas que se producían y deterioraban la vida económica y social. Sin embargo, la normativa dictada en esta materia solía quedar en letra muerta, de escasa o nula aplicación por la escasez de medios (mecanismos de inspección) destinados a garantizar la observancia de los preceptos. A finales de la segunda década del siglo xx, la mayor parte de países con industria culminaban con la aprobación de leyes análogas que limitaban la jornada a un máximo de ocho horas diarias y cuarenta y ocho semanales. Hasta aquí podemos decir que la reducción de jornada constituye un objetivo que es alcanzar el derecho al descanso ${ }^{3}$.

\footnotetext{
2 R. JÁuregui, F. Egea y J. DE la Puerta, El tiempo que vivimos y el reparto de trabajo. La gran transformación del trabajo, la jornada laboral y el tiempo libre, Barcelona, Paidós, 1998, pp. 168-169.

3 M. C. Palomeque López, Derecho del trabajo e ideología, 7. a ed. revisada, Madrid, Tecnos, 2011, pp. 33-34.
} 


\section{EL PROBLEMA DE LIMITAR LA JORNADA DE TRABAJO}

Llegados a este punto y al analizar el problema de la jornada de trabajo nos surge una cuestión, ¿isigue siendo las ocho horas de trabajo al día el límite normal de la jornada de trabajo? Este límite fue estudiado por un gran número de autores ${ }^{4}$ teniendo en cuenta la esperanza de vida en el siglo XIX y el tiempo de trabajo total (años) de un obrero. En los momentos actuales todas las condiciones han variado: la esperanza de vida ha aumentado y el tiempo normal de trabajo de un obrero se prolonga a los cuarenta años. A pesar del cambio significativo en esta proporción, el límite normal de la jornada de trabajo se ha mantenido en ocho horas.

La lucha de la clase obrera por la jornada laboral de ocho horas ha pasado por varias etapas. La tercera va desde el final de la Primera Guerra Mundial hasta los años ochenta. En la posguerra la presión de las organizaciones de trabajadores aceleró el progreso hacia la jornada de ocho horas. El resultado del mismo fue que la limitación de la duración de trabajo por la legislación de ocho horas por día o cuarenta y ocho semanales se llevó a la práctica, en algunas ocupaciones o ramas de la industria, de la mayoría de los Estados.

En 1919, a raíz del sistema de Tratados de la Paz de Versalles, se funda la Organización Internacional del Trabajo, que ya desde su fundación ha atendido la elaboración y adopción de normas internacionales sobre las horas de trabajo. En el Preámbulo de la Parte XIII la OIT previó expresamente «la reglamentación de las horas de trabajo, fijación de la duración máxima de la jornada y de la semana de trabajo» entre las medidas imprescindibles para mejorar las condiciones de trabajo. Al mismo tiempo, la adopción de la jornada de ocho horas o la semana de cuarenta y ocho horas como la norma a la que se debía aspirar en los casos en que todavía no se había conseguido. Con esta reglamentación la OIT logra que la adopción de la jornada de ocho horas se instituyera como norma internacional respondiendo a una de las principales demandas formuladas por las organizaciones de trabajadores.

En la primera reunión de la Conferencia Internacional del Trabajo se adoptó el Convenio sobre las horas de trabajo (industria), 1919, núm. $1^{5}$.

${ }^{4}$ Entre ellos K. MARX, «Trabajo asalariado y capital», disponible en www.geocities.com/ CapitolHill/Lobby/3554.

5 R. Jáuregui, F. Egea y J. de la Puerta, op. cit., pp. 180-181. 
Aun cuando diversos países ya habían introducido la jornada de ocho horas y la semana de cuarenta y ocho horas en su legislación antes de la adopción del Convenio núm. 1, surgieron dificultades para ratificar este instrumento, sobre todo por parte de los países más industrializados. Pero a pesar de ello, esto supuso una evolución de la legislación laboral de muchos países en materia de tiempo de trabajo. Otro paso importante lo constituyó la aprobación del Convenio sobre las horas de trabajo en comercio y oficinas de 1930, Convenio núm. 30. La significación del mismo radica en su objetivo: hacer extensivas las disposiciones relativas a las horas de trabajo a todos los trabajadores. Posteriormente fueron adoptados otros convenios relacionados con la jornada de ocho horas y su reglamentación. De suma importancia es el Convenio núm. 47 de 1935 sobre las cuarenta horas, aplicado de tal forma que no implique una disminución del nivel de vida de los trabajadores. A pesar de la importancia de la cuestión de la duración del trabajo, la tasa de ratificación de los Convenios núms. 1 y 30 puede calificarse tan sólo de modesta.

Los últimos datos que he encontrado sobre la ratificación de dichos Convenios son de $2012^{6}$. El Convenio núm. 1 ha sido ratificado por cincuenta y dos Estados miembros, registrándose la última ratificación el 14 de junio de 1988 por Guatemala y con una denuncia por parte de Nueva Zelanda el 29 de marzo de 1939, y el Convenio núm. 30 fue ratificado por treinta Estados miembros, siendo la ratificación más reciente del 12 de junio de 1985 por Guinea Ecuatorial, que a su vez ha sido denunciado por dos países, Finlandia y Nueva Zelanda. Además de ello, de los diez Estados miembros de mayor importancia industrial de la OIT no han ratificado el Convenio núm. 30. Los pretextos son alegar que las disposiciones de la OIT frenaban las tareas de reconstrucción de la posguerra y que si no se acataban por el pleno de los países se afectaban las posiciones competitivas de los firmantes ${ }^{7}$.

\section{EL PROBLEMA DEL EMPLEO}

Ante la gravedad del problema de desempleo en Europa, en los años noventa tuvieron lugar iniciativas y propuestas nacionales que lograron

\footnotetext{
${ }^{6}$ Fuente de la OIT, normas de trabajo, ratificación por Convenios, página web: www.ilo. org/dyn/normlex/es.

7 J. J. GonZÁlez SÁnCHeZ, «La política social europea. Nuevas tendencias para la lucha contra el desempleo: sobre la reducción del tiempo de trabajo. OIT y mundialización de la economía», Tribuna Social. Revista de Seguridad Social y Laboral, núm. 88 (1998), pp. 35-52.
} 
el debate en torno a los métodos de lucha contra el paro. Las tasas de desempleo en esos momentos alcanzaban en el conjunto de la UE un 11 por 100 y en España un 20 por 100 (situación similar a la actual), afectando a colectivos como mujeres, jóvenes y parados de larga duración. Entre las causas de este incremento notable de paro los expertos coincidían en lo siguiente: a) falta de adecuación de la oferta educativa para los jóvenes y la modernización tecnológica que originó el denominado «paro tecnológico excesivo» ${ }^{8}$; b) la incorporación de la mujer al mercado de trabajo ha ejercido una mayor presión sobre éste y ha contribuido a elevar el índice de paro; c) la evolución de los costes laborales unitarios en España no se ha visto acompañada de una mejora semejante de la productividad. Ante esta situación se realizaron propuestas de lucha contra el desempleo en los años noventa desde la UE y desde los países miembros, sin embargo, todas las propuestas partían de que el crecimiento económico por sí solo no era suficiente para crear empleo, por lo que eran necesarias otras medidas, como nuevas iniciativas que supusieran una postura activa de lucha contra el paro. Entre estas medidas se encontraban: a) la propuesta de reducción del tiempo de trabajo llevada a cabo de forma progresiva y mediante la negociación colectiva por sectores y empresas; $b$ ) la disminución, hasta la supresión, de las horas extraordinarias y compensación por descanso o acumulación de cara a una posible jubilación anticipada; c) reestructuración del sistema de desempleo?

La regulación europea más reciente sobre el tiempo de trabajo se contiene en la Directiva 2003/88/CE del Parlamento Europeo y del Consejo, de 4 de noviembre de 2003 , relativa a determinados aspectos de la ordenación del tiempo de trabajo, que contiene las condiciones mínimas que han de aplicar los distintos Estados miembros y trata de lograr un equilibrio entre el objetivo principal que es la seguridad y salud de los trabajadores y también responder a las necesidades de una economía europea moderna.

${ }^{8}$ La tecnología moderna es considerada como la causa generadora del paro laboral en Europa. La tecnología sustituye al trabajo, de tal suerte que para producir lo mismo se necesita menos trabajo. Así la cantidad de trabajo necesario desciende y la sociedad debería repartir el trabajo disponible.

9 En el sentido de reducir las cuotas de empresarios y trabajadores en los contratos estables, incremento de las cuotas de contratos temporales como medida disuasoria de este tipo de contratación o la bajada de las cuotas sociales de las primeras treinta y cinco horas e incremento considerable a partir del resto. 


\section{VISIÓN NACIONAL DE LA REDUCCIÓN DEL TIEMPO DE TRABAJO}

En España el tema del tiempo de trabajo como medida de reparto del trabajo se ha quedado en el debate sin ir más allá de simples planteamientos. Este recurso apareció en los años ochenta y se vuelve a replantear en momentos de crisis.

En la Constitución Española (CE) se puede advertir que se puede ir en dirección hacia la reducción de la jornada de trabajo, aunque también se imponen ciertas condiciones. Pues bien, tanto el derecho al trabajo como a la libre elección de profesión u oficio se muestran como un espacio a la libertad de la persona que forma parte del contenido esencial de tal derecho. De modo que este contenido esencial no puede ser negado, salvo que entre en colisión con otros valores circunstanciales de transcendencia mayor o equiparable que justifiquen restringir la libertad individual.

Desde la doctrina mayoritaria española más autorizada, y en consonancia con los textos internacionales, se sugiere una interpretación del art. 10.2 CE donde se considera el derecho al trabajo no como un valor absoluto, sino como un espacio jurídico que limita y puede ser limitado por otros derechos y libertades; por ello no es posible ofrecer garantías jurídicas plenas de satisfacción del derecho al trabajo, y es que la libertad de empresa en una economía de mercado (art. $38 \mathrm{CE}$ ), y en menor medida el derecho a la propiedad privada (art. $33 \mathrm{CE}$ ), descartan la posibilidad económica de que los poderes públicos satisfagan sin más el derecho al trabajo, o lo que es igual, que «la determinación del volumen, cualidad y circunstancias de la oferta de trabajo está en función de decisiones económicas privadas». La libertad de empresa no se corresponde con un deber del empresario de crear empleo, ya que la libertad de la que se deriva la empresa es un derecho subjetivo.

Por lo que aquí interesa, la libertad de gestión de la unidad productiva de la que se deriva la contratación de trabajadores sí puede ser sometida por ley a ciertos límites (art. 53.1 CE). Por lo que habrá que buscar aquellos mecanismos limitativos de la libertad de empresa que permitan al Estado poner en marcha políticas económicas diversas destinadas a la creación de empleo como forma de hacer efectivo el derecho al trabajo. De tal forma que la política orientada al pleno empleo (art. $40 \mathrm{CE}$ ), así como el derecho al trabajo (art. 35.1 CE), están relacionados. Es esta concepción del derecho al trabajo la que justificaría la intervención de los pode- 
res públicos para favorecer el reparto de trabajo mediante la reducción legal de la jornada; así la idea de la disminución de la jornada de trabajo que tenía como fin la seguridad, la salud y el descanso es completada en la actualidad por la reducción de jornada para crear empleo ${ }^{10}$.

La ordenación del tiempo de trabajo y las normas que lo conforman constituye un factor fundamental para posibilitar un mejor reparto del trabajo y facilitar nuevas colocaciones. En esta dirección incidió la Directiva 93/104 del Consejo de la UE y la Ley 11/1994, de 19 de mayo, incorporada a los arts. 34 y sigs. del Texto Refundido de la Ley de los Trabajadores (RDL 1/1995, de 24 de marzo). En la Exposición de Motivos de aquella Ley se señalaba cómo confluyen en esta materia elementos propios de la política de empleo, perceptibles en la actuación sobre la jornada de trabajo desde criterios de reparto del volumen del empleo, pero también en la construcción de un marco jurídico que posibilite la máxima eficacia del proceso productivo ${ }^{11}$.

La regulación de la jornada laboral en el Estatuto de los Trabajadores favorece la idea de gestionar de manera más flexible los recursos humanos de la empresa y reducir el tiempo de trabajo mediante negociación colectiva en aquellos supuestos considerados por los agentes sociales económicamente viables. Sin embargo es conveniente señalar las modificaciones sufridas, en cuanto a la negociación colectiva, mediante el art. 14 del RDL 3/2012, donde se establece que cuando concurran causas técnicas, económicas o de producción, mediante acuerdo entre empresa y los representantes de los trabajadores legitimados para negociar un convenio, se podrá proceder a inaplicar en la empresa las condiciones de trabajo previstas en el convenio colectivo aplicable, entre esas materias está la jornada de trabajo. De tal forma que la autonomía colectiva queda relegada a un segundo plano. Así, la autonomía colectiva en estos casos no tiene la suficiente fuerza para imponer sus pautas y sería entonces necesaria la regulación mediante ley. Con esta idea quiero manifestar que la autonomía colectiva puede resultar adecuada cuando por sí sola tiene fuerza imperante, pero si se le desgaja esa fuerza entonces la reducción de la jornada de trabajo, para cumplir el objetivo del reparto de trabajo, requiere que sea regulado mediante ley, pues la negocia-

10 A. MarTín VALVERDE, «Derecho al trabajo, pleno empleo y deber de trabajar», en VVAA, Derecho del trabajo y de la seguridad social en la Constitución, Madrid, CEC, 1980, pp. 180 y ss.

${ }^{11}$ J. I. García Ninet, «La ordenación del tiempo de trabajo», Revista Tribuna Social, número especial (1995). 
ción colectiva y su correspondiente regulación estatal no facilitan el efectivo cumplimiento.

\section{EXPERIENCIA EN FRANCIA}

El tema de la reducción del tiempo de trabajo como medida de creación de empleo ha sido objeto de diálogo y estudio, pero no ha sido llevado a la práctica desde las instituciones europeas. Algunos países sí han llevado a cabo la medida de reducción de jornada como mecanismo de creación de empleo, concretamente en Francia a principios de los ochenta. El gobierno francés implementó un protocolo con la Ordenanza de 16 de enero de 1982 que introdujo la quinta semana de vacaciones y redujo la semana laboral a treinta y nueve horas. Las implicaciones de esta medida hay que analizarlas a la luz del contexto general de Francia. Pues bien, en esos momentos en Francia tras el fracaso del «keynesianismo» se tuvieron que llevar a cabo políticas monetarias y fiscales restrictivas y acudir a criterios de competitividad basados en la contención de costes, y esa situación alteraba el marco en el que el proceso de reducción de tiempo de trabajo tenía sentido, es decir, la reducción del tiempo de trabajo debía propiciarse con una política de crecimiento, para así incrementar su intensidad creadora de empleo. Al no ser así, lo que ocurrió fue que no se crearon empleos y se elevaron los costes laborales unitarios ${ }^{12}$. Pero Francia abandonaba su concepción de reducción de la jornada de trabajo al aprobar la Ley Seguin, haciendo posible la modulación del tiempo de trabajo por acuerdo sectorial, de rama o de empresa. Lo que se produjo fue una flexibilización de la jornada, mientras que la reducción de la jornada caía en el olvido.

Años más tarde, en 1996, se aprobó por el Parlamento francés la Ley Robin, que establecía una jornada laboral de treinta y dos horas semanales y una reducción del salario del 5 por 100, lo que estimaban que crearía un 10 por 100 de empleo. Para compensar la pérdida de salarios proponía que las empresas introdujeran planes de participación en los incrementos de la productividad ${ }^{13}$.

12 R. JÁuregui, F. Egea y J. DE la Puerta, «La gran transformación: la jornada laboral, el tiempo en la estrategia para el empleo y el desarrollo sostenible», El tiempo que vivimos y el reparto del trabajo. La gran transformación del trabajo, la jornada laboral y el tiempo libre, Barcelona, Paidós, 1998, pp. 205-206.

${ }_{13}$ Esta ley fue criticada por algunos expertos, pues consideraban que a largo plazo lo que conseguirían las empresas sería introducir un amplio margen de flexibilidad de libre 
En octubre de 1997 surgió la Ley Aubry, implementando la jornada de treinta y cinco horas semanales a partir del año 2000. El apoyo financiero ofrecía ayudas de 9.000 francos por trabajador a todas las empresas que negociasen con los sindicatos una reducción del tiempo de trabajo de como mínimo un 10 por 100 y a su vez aumentaran un 6 por 100 su plantilla. Las horas trabajadas por encima de las treinta y cinco horas semanales se considerarían extraordinarias y penalizadas. Esta ley fue sustituida por la Ley Aubry II, relativa a la reducción negociada del tiempo de trabajo, cuyo objetivo era confirmar el objetivo de la ley anterior ${ }^{14}$. Pero a partir del 2002 en Francia se impone como objetivo primordial la consigna de «libertad de trabajar más para ganar más», acabando así con los años anteriores de reducir el tiempo de trabajo.

La experiencia en Francia de la reducción de los tiempos de trabajo no fue la esperada, es decir, no se creó empleo. Los defensores de la reducción de la jornada de trabajo lo justificaron afirmando que no se dieron los factores adecuados para que se produjeran los resultados esperados. Dicha medida se debía llevar a cabo bajo unas circunstancias específicas, como encontrarse en una fase de crecimiento económico, lo cual no fue así, y desembocó en una medida errónea para crear empleo.

\section{DINÁMICA DE INTERCAMBIO TRABAJO Y SALARIO}

Como he expuesto anteriormente, en épocas de recesión económica y desempleo se ha propuesto a menudo reducir la jornada de trabajo. Pero esta reducción hay que distinguirla de la repartición del trabajo como sistema provisional acordado entre los trabajadores de una empresa o establecimiento en respuesta a una disminución de la actividad de los mismos. En tales casos, para evitar los despidos los trabajadores aceptan una jornada semanal más corta y un menor salario, es decir, aceptan un trabajo de jornada reducida. En algunos países se está fomentando esta solución, así es el caso de Alemania y España. En estos casos el sistema se limita a un corto periodo de tiempo y se sustituye por la jornada y salarios normales en el momento en que la empresa supera las dificultades, o por una

\footnotetext{
negociación entre sindicatos y empresarios, y quien tiene más poder es la empresa, por lo que finalmente esto daría lugar a una flexibilidad al gusto del empresario.

${ }^{14}$ E. Martín Puebla, La reducción del tiempo de trabajo en Francia: un análisis desde el Derecho, Informes y Estudios, Relaciones Laborales, Salamanca, Ministerio de Trabajo y Asuntos Sociales, 2006, pp. 36-38.
} 
reducción de la plantilla y la consiguiente reanudación de la jornada normal si se prolonga el problema de la empresa. Este tipo de reducción de jornada, con la consiguiente reducción de los salarios, no es bien recibida en muchos casos por los trabajadores, pues supone una minoración de los ingresos, pero entre quedarse sin empleo o sufrir esta medida prefieren que se les aplique lo último.

La reducción de la jornada de trabajo sin la consiguiente disminución de los salarios es una medida que obviamente no consta con la oposición de los trabajadores, pero sí de la empresa, pues supone en principio una subida de los costes laborales. Los argumentos que sirven de base para sostener que la reducción de la jornada reduciría el desempleo son de dos tipos: uno relativo al bienestar social y el otro a la igualdad distributiva. Desde el punto de vista del bienestar social, quienes tienen un trabajo se beneficiarían de la reducción del tiempo de trabajo mediante una mejora de su calidad de vida y quienes escapan del desempleo mejorarían su situación económica al tener un trabajo. El desempleo se puede considerar una forma de distribución desigual, en la que una minoría está privada de trabajo; la reducción de la jornada permitiría así distribuir el trabajo de forma más igualitaria. Sin embargo, estos argumentos a favor encuentran un obstáculo importante y es que para las empresas supone un aumento de los costes laborales. Ante este inconveniente se han manifestado algunos estudios empíricos que muestran que la reducción de jornada puede aumentar la productividad de las personas, siempre que se reorganice el proceso de trabajo de forma adecuada, y con ello se mantendrían los beneficios empresariales.

Además, la relación más importante entre jornada de trabajo y producción se da a nivel fisiológico. La gente es capaz de trabajar durante periodos continuados considerablemente largos y durante un porcentaje bastante alto de su jornada diaria total, pero la adaptación a las jornadas de trabajo prolongadas se realiza mediante un ajuste del ritmo o la intensidad del trabajo, haciendo más lentos los movimientos e intercalando mayor número de pausas entre ellos.

Como vemos, la gran oposición a la reducción del tiempo de trabajo se debe a los aumentos de los costes laborales para las empresas. Pero en la medida que dicha reducción venga acompañada de una serie de políticas y formas de reorganizar el proceso de producción y se demuestre que un menor tiempo de trabajo puede aumentar la productividad, esta reducción de jornada puede ser valorada positivamente. 


\section{CONCLUSIONES}

Más allá de estas consideraciones sobre el tiempo de trabajo la propuesta de reducción del tiempo de trabajo se plantea como una vía de reparto de la productividad generada durante las últimas décadas hacia el empleo. El tiempo de trabajo y su regulación se configuran como una pieza importante de la política de empleo, pero que debe actuar en estrecha relación con otras, como, por ejemplo, con los nuevos yacimientos del empleo. Además es necesario abordar la reducción de la jornada laboral teniendo en cuenta algunos aspectos característicos del mercado de trabajo en la actualidad. Así, en el caso español se puede comprobar el elevado número de horas extraordinarias que se registran legalmente, a las que se suman las horas extraordinarias no registradas por las estadísticas y en muchos casos ni siquiera retribuidas a los trabajadores. ¿Es compatible una reducción significativa del tiempo de trabajo si no se actúa sobre las horas extraordinarias?

A este interrogante se le pueden sumar otros relacionados con la creciente individualización de las relaciones laborales en algunos sectores y colectivos de trabajadores para los que la prolongación de jornada o la disponibilidad son situaciones frecuentes. En relación con esto también cabe reflexionar sobre el contrato a tiempo parcial, dado que su jornada reducida puede ser una importante vía de inserción en el mercado de trabajo para colectivos como jóvenes o mujeres, con necesidades sociales específicas, y ser una vía importante de creación de empleo de calidad, siempre y cuando los contratos sea de carácter voluntario y no supongan una merma de los niveles estándares de protección social de los contratos a tiempo completo.

La crisis del empleo es un proceso social de gran complejidad que requiere adoptar diversas perspectivas e instrumentos que permitan incidir desde el ámbito internacional hasta los ámbitos locales. La conclusión es que un problema complejo no acepta soluciones sencillas ni únicas. Por ello, la propuesta de reducción del tiempo de trabajo tiene valor social si se considera la propia diversidad de situaciones en las que se puede plasmar y los distintos colectivos sociales a los que se puede favorecer. Esta orientación requiere adoptar una perspectiva internacional, aunque el punto de partida sea la acción a nivel nacional. Desde esta perspectiva, cabe apoyar la reducción de la jornada laboral, desarrollada sectorialmente a través de la negociación colectiva y que cuente con un marco legal que estimule su desarrollo. 